\title{
Computational Study on Trabecular Bone Remodeling in Human Femur under Reduced Weight-bearing Conditions*
}

\author{
Jiyean KWON**, Hisashi NAITO**, Takeshi MATSUMOTO** \\ and Masao TANAKA** \\ **Department of Mechanical Science and Bioengineering, Osaka University, \\ 1-3 Machikaneyama, Toyonaka, Osaka, 560-8531, Japan \\ E-mail: [lilie, naito, matsu, tanaka]@me.es.osaka-u.ac.jp
}

\begin{abstract}
Trabecular bone structure is determined by a balance between osteoblastic bone formation and osteoclastic bone resorption, which is regulated partly by osteocytes according to their mechanical environments. There have been a number of studies on bone remodeling in response to mechanical stimuli, mainly in the physiological range. This study uses a mathematical model previously formulated for surface remodeling available even for disuse and overuse ranges considering osteocyte apoptosis and targeted remodeling. Thus, the present model allows exhibiting the changes of trabecular bone structure under, below, and beyond the daily loading condition. In this study, we carried out computer simulation of bone remodeling in human femur under normal daily loading condition and reduced weight-bearing conditions (infrequent and cane-assisted walking conditions). Decreased trabecular bone with reducing loading condition was shown, and the trabecular bone structure at various degrees of disuse was consistent to Singh Index for osteoporosis diagnosis.
\end{abstract}

Key words: Biomechanics, Bone Remodeling, Disuse, Trabecular Structure, Osteoporotic Femur

\section{Introduction}

Trabecular bone structure is continuously renewed by remodeling, that is, the alternately-repeated events of local bone resorption and formation. Their imbalance, leading to bone loss or gain, is under the influence of mechanical stimuli on trabeculae ${ }^{(1) \sim(4)}$. Therefore, trabecular bone structure varies in a manner dependent on the magnitude, direction, and frequency of skeletal loading.

Reduced skeletal loading causes trabecular bone loss in humans and other animals. Skeletal unloading during space flight resulted in bone loss ${ }^{(5),(6)}$, and the unloading-induced deficit in bone mineral density was observed in human bed rest studies ${ }^{(7)} \sim(9)$. In postmenopausal woman, the statistics analysis showed the correlation between body mass index and osteoporosis ${ }^{(10),(11)}$. The experimental and simulation results showed that tail suspension induces reduced bone formation in rat tibiae ${ }^{(12) ~} \sim(14)$. Furthermore, the predominant effect of unloading was found to be the down-regulation of osteoblast activity possibly with the up-regulation of osteoclast activity in canine studies ${ }^{(15) \sim(16)}$. It is clear from these studies that reduced skeletal unloading causes bone loss through the imbalance between bone formation and resorption.

${ }^{*}$ Received 30 July, 2010 (No. 10-0318) [DOI: 10.1299/jbse.5.552]

Copyright (C) 2010 by JSME 
Mechanostat theory gives us a basic frame work to grasp the mechanical adaptation by bone remodeling ${ }^{(17)}$. Computational studies of bone remodeling have been carried out for quantitative predictions of bone formation and resorption on the basis of this theory ${ }^{(18) \sim(25)}$. However, in those studies, the attention has been focused mainly on the physiological range of mechanical stimuli called "the physiological window (PW)". Because the mechanical stimuli to bone are not limited only to the physiological range, it is worth considering a mathematical model of bone remodeling available over the wide range of mechanical stimuli.

In this study, to investigate trabecular bone loss and structure changes under reduced weight-bearing conditions, we use a surface remodeling model proposed in the previous report ${ }^{(26)}$. In this model, based on the mechanostat theory, we assumed that in a low strain range (disuse window, DW), bone resorption occurs at an accelerated rate with the decrease in strain owing to low strain-induced osteocyte apoptosis and that in a high strain range (overuse window, OW), bone formation occurs at an accelerated rate with the increase in strain (targeted remodeling). In PW, bone formation or resorption was assumed to occur stochastically according to the degree of local stress non-uniformity ${ }^{(21)}$. Using this model, we carried out the computer simulation for trabecular bone remodeling in human femur under the conditions of bed rest, decreased walking, and cane-assisted walking.

\section{Methods}

Trabecular structure was discretized using voxel finite elements, as shown in Fig. 1. Trabecular resorption/formation is expressed by removing/adding voxel elements referring to the equivalent strain $(\bar{\varepsilon})$ estimated by finite element analysis. In the present model of trabecular surface remodeling, we considered three windows, PW, DW, and OW, which were distinguished by the threshold value of equivalent strain: DW for $\bar{\varepsilon}<\varepsilon_{d u}$, PW for $\varepsilon_{p l}$ $<\bar{\varepsilon}<\varepsilon_{p u}$, and OW for $\bar{\varepsilon}>\varepsilon_{o l}$ (Fig. 2). The quantity of removing/adding trabecular elements is calculated by activation frequency and probability of bone resorption/formation. We set the rules for bone resorption and formation in each window. Outline of the surface remodeling model used here is described in the following section.

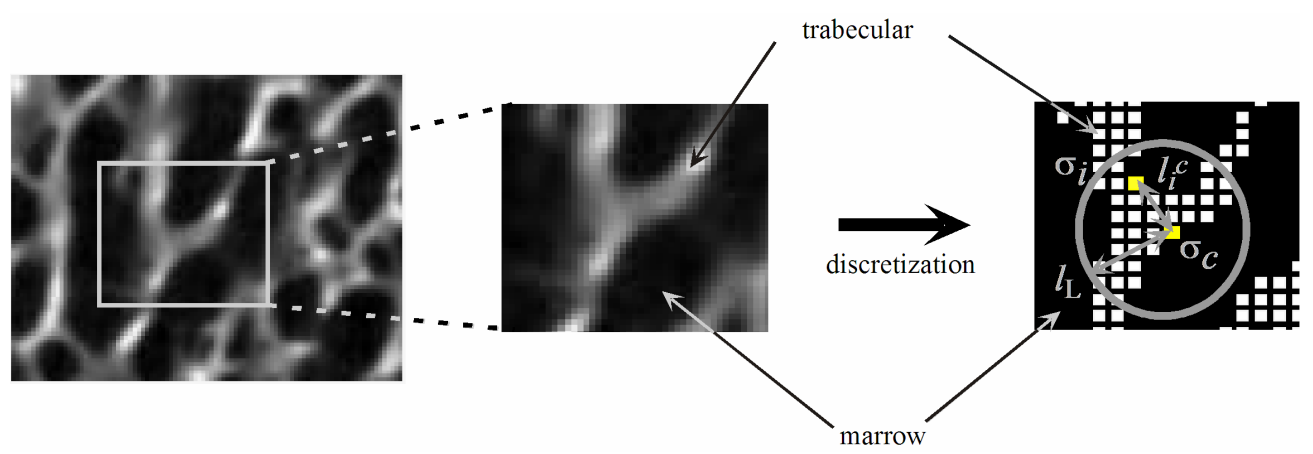

Fig. 1 Discretized trabecular bone.

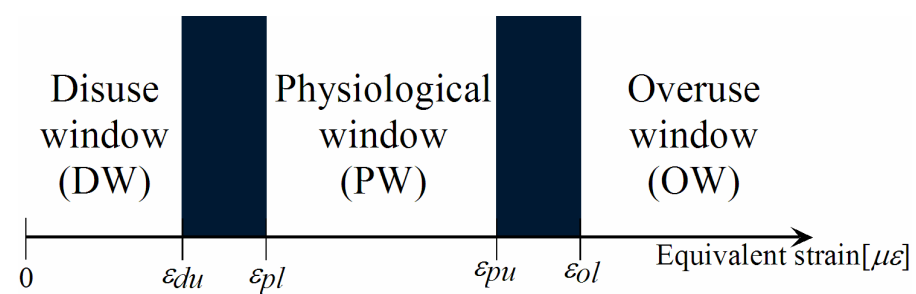

Fig. 2 Windows categorized by the equivalent strain and transition regions between windows. 


\subsection{Mathematical model for surface remodeling}

In PW of $\varepsilon_{p l}<\bar{\varepsilon}<\varepsilon_{p u}$, the non-uniformity of the stress/strain distribution was taken as driving stimuli ${ }^{(21) \sim(25)}$. For the voxel representing a point $c$ on bone surface, the degree of local stress non-uniformity $\Gamma_{c}$ was quantified as

$$
\Gamma_{c}=\ln \left(\sigma_{c} \sum_{i}^{N} w\left(l_{i}^{c}\right) / \sum_{i}^{N} w\left(l_{i}^{c}\right) \sigma_{i}\right)
$$

where $\sigma_{c}$ denotes the stress at the point $c$ and $\sigma_{i}$ denotes the representative stress at a point $i$ with the distance $l_{i}^{c}$ apart from $c$. The weight function $w\left(l_{i}^{c}\right)\left[w\left(l_{i}^{c}\right)>0\left(0 \leq l_{i}^{c}<l_{L}\right)\right]$ decays with distance, within the sensing distance $l_{L}$. The probability of bone resorption or formation at the point $c$ is determined by $f\left(\Gamma_{\mathrm{c}}\right)$, which is illustrated in Fig. 3, where $\Gamma_{u}$ and $\Gamma_{l}$ denote the lower and upper thresholds, respectively. When $f\left(\Gamma_{c}\right)=1$, bone formation occurs, resulting in the addition of voxel elements added around the surface point $c$, and when $f\left(\Gamma_{c}\right)=-1$, bone resorption occurs, resulting in the subtraction of voxel $c$. No change occurs at and around $c$ when $f\left(\Gamma_{c}\right)=0$. Moreover, in the cases of $0<\Gamma_{c} \leq \Gamma_{u}$ and $\Gamma_{l} \leq \Gamma_{c}<0$, bone formation and resorption occurs stochastically according to probability $f\left(\Gamma_{c}\right)$. In this study, one-simulation step time was assumed to correspond to a bone turn-over period in PW; that is, one process of voxel removal or addition works in one simulation step in PW. Here, activation frequencies for bone resorption $\left(A F_{r}\right)$ and formation $\left(A F_{f}\right)$ were defined as turn-over frequency for bone resorption and formation per simulation step, respectively, i.e., relative rate for bone resorption and formation based on those in $\mathrm{PW}\left(A F_{f}=A F_{r}=1\right.$ in $\left.\mathrm{PW}\right)$.

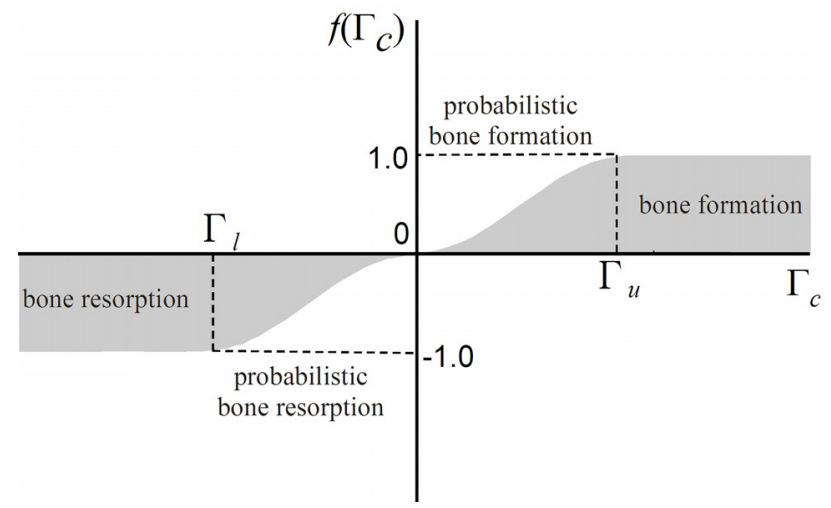

Fig. 3 Determinant function of bone resorption and formation in PW.

In DW of $\bar{\varepsilon}<\varepsilon_{d u}$, only bone resorption becomes dominant, and the activation frequency of resorption becomes larger as the strain becomes smaller due to the osteocyte apoptosis. Thus, the probability of bone resorption is unity, i.e., $\mathrm{P}_{\mathrm{DW}}=1$ and $f\left(\Gamma_{c}\right)=-1$. The activation frequency for bone resorption $A F_{r}$ increases exponentially with the decrease of strain at point $c\left(\varepsilon_{c}\right)$ and reaches saturation $\left(A F_{r}=A F_{r \max }\right)$ when $\varepsilon_{c}=\varepsilon_{\min }$ (Fig. 4(a) $)^{(27) \sim(29)}$.

In OW of $\varepsilon_{o l}<\bar{\varepsilon}$, bone formation via targeted remodeling has been reported ${ }^{(30),(31)}$. The probability of bone formation as the result of remodeling turnover is unity, i.e., $\mathrm{P}_{\mathrm{OW}}=1$ and $f\left(\Gamma_{c}\right)=1$. The activation frequency for bone formation $A F_{f}$, assumed by a sigmoid function defined over the strain range of $\varepsilon_{o l}$ to $\varepsilon_{\max }$ (Fig. 4(b)), increases with $\varepsilon_{c}$ and reaches saturation $\left(A F_{r}=A F_{\text {fmax }}\right)$ when $\varepsilon_{c}=\varepsilon_{\max }$ (Fig. 4(b)). It is noted that the pathologically large mechanical stimuli is not considered here.

Mathematical models of bone remodeling established in DW, PW, and OW were integrated by introducing transition regions ${ }^{(26)}$. In the transition region $\varepsilon_{d u}<\bar{\varepsilon}<\varepsilon_{p l}$ between DW and PW or the transition region $\varepsilon_{p u}<\bar{\varepsilon}<\varepsilon_{o l}$ between PW and OW, the probability of bone resorption $P_{r}{ }^{*}$ or formation $P_{f}^{*}$ was given by a linearly weighed sum of $-\mathrm{P}_{\mathrm{DW}}$ or $\mathrm{P}_{\mathrm{OW}}$ 
and $f\left(\Gamma_{c}\right)$. The resorption and formulation probabilities and the threshold values for remodeling simulation are summarized in Eqs. (2)-(4) and Table 1, respectively.

$$
\begin{aligned}
& f^{*}\left(\Gamma_{c}, \bar{\varepsilon}_{c}\right)= \begin{cases}-\mathrm{P}_{\mathrm{DW}} & \left(\bar{\varepsilon}_{c}<\varepsilon_{d u}\right) \\
-\left(1-\frac{\overline{\varepsilon_{c}}-\varepsilon_{d u}}{\varepsilon_{p l}-\varepsilon_{d u}}\right) P_{D W}+\frac{\bar{\varepsilon}_{c}-\varepsilon_{d u}}{\varepsilon_{p l}-\varepsilon_{d u}} f\left(\Gamma_{c}\right) & \left(\varepsilon_{d u} \leq \bar{\varepsilon}_{c}<\varepsilon_{p l}\right) \\
f\left(\Gamma_{c}\right) & \left(\varepsilon_{p l} \leq \bar{\varepsilon}_{c}<\varepsilon_{p u}\right) \\
\left(1-\frac{\varepsilon_{c}-\varepsilon_{p u}}{\varepsilon_{o l}-\varepsilon_{p u}}\right) f\left(\Gamma_{c}\right)+\frac{\varepsilon_{c}-\varepsilon_{p u}}{\varepsilon_{o l}-\varepsilon_{p u}} P_{O W} & \left(\varepsilon_{p u} \leq \bar{\varepsilon}_{c}<\varepsilon_{o l}\right) \\
\mathrm{P}_{\mathrm{OW}} & \left(\varepsilon_{o l} \leq \bar{\varepsilon}_{c}\right)\end{cases} \\
& P_{f} *\left(\Gamma_{c}, \bar{\varepsilon}_{c}\right)=f^{*}\left(\Gamma_{c}, \bar{\varepsilon}_{c}\right) \quad\left(f^{*}\left(\Gamma_{c}, \bar{\varepsilon}_{c}\right) \geq 0\right) \\
& P_{r}^{*}\left(\Gamma_{c}, \bar{\varepsilon}_{c}\right)=\left|f^{*}\left(\Gamma_{c}, \bar{\varepsilon}_{c}\right)\right| \quad\left(f^{*}\left(\Gamma_{c}, \bar{\varepsilon}_{c}\right)<0\right)
\end{aligned}
$$

Using this integrated model, trabecular bone structure was simulated by the following procedures.

(1) The initial shape of the trabecular bone is discretized by voxel finite elements and Young's modulus and Poisson's ratio are given.

(2) The equivalent stress and strain distributions are determined under a given boundary condition.

(3) The probabilities $P_{f}^{*}$ and $P_{r}^{*}$ of bone formation or resorption are calculated for every voxel on bone surface by Eqs. (2)-(4).

(4) The activation frequency $A F_{f}$ and $A F_{r}$ of bone formation or resorption are calculated for every voxel on bone surface. These are rounded to the nearest integer number and truncated by $A F_{\text {fmax }}$ or $A F_{r \max }$.

(5) Bone surface movement (voxel removal or addition) is determined stochastically based on $P_{f}^{*}$ and $P_{r}^{*}$.

(6) For voxels with activation frequency $A F_{r}$ or $A F_{f}$ larger than one, the same surface movements in procedure (5) are repeated $A F_{r}$ or $A F_{f}$ times.

(7) Procedures (2)-(6) are repeated until equilibrium is achieved.

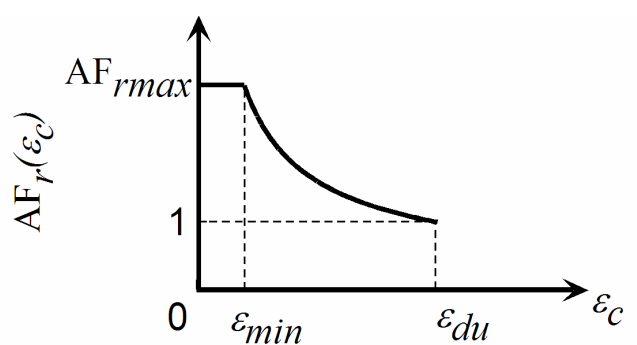

(a) Activation frequency in DW



(b) Activation frequency in $\mathrm{OW}$

Fig. 4 Activation frequency as a function of equivalent strain for DW and $\mathrm{OW}$ windows. (It is noted that activation frequency is rounded to the nearest integer number in simulation.) 


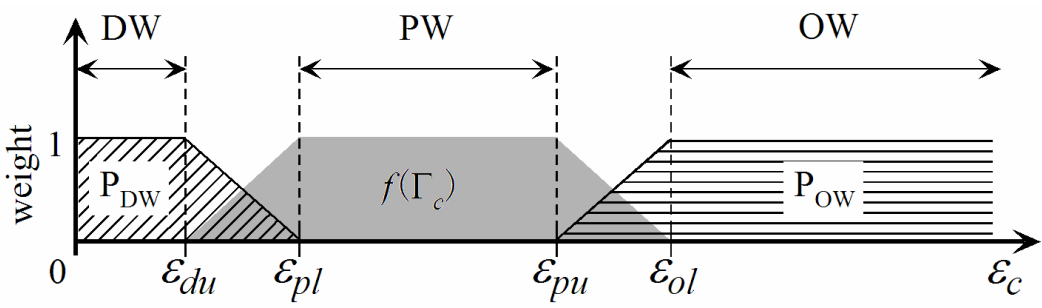

Fig. 5 Integrated probability of remodeling.

Table 1 Simulation parameters.

\begin{tabular}{c|c|c|c}
\hline$\varepsilon_{\min }$ & $20[\mu \varepsilon]^{(17)}$ & $\varepsilon_{\max }$ & $10000[\mu \varepsilon]^{(17)}$ \\
$\varepsilon_{d u}$ & $50[\mu \varepsilon]^{(17)}$ & $\mathrm{AF}_{r \max }$ & $3^{(29)}$ \\
$\varepsilon_{p l}$ & $100[\mu \varepsilon]^{(17)}$ & $\mathrm{AF}_{f \max }$ & $3^{(31)}$ \\
$\varepsilon_{p u}$ & $1000[\mu \varepsilon]^{(17)}$ & $\Gamma_{l}$ & $-1^{(21)}$ \\
$\varepsilon_{o l}$ & $2500[\mu \varepsilon]^{(17)}$ & $\Gamma_{u}$ & $1^{(21)}$ \\
\hline
\end{tabular}

\subsection{Finite element model of human proximal femur}

The three-dimensional profile of human proximal femur is reconstructed using computer tomography (female, 70 years, hip osteoarthritis), the resolution of the images was $0.7 \mathrm{~mm}$ so that the entire volume could be captured with slice image of $512 \times 512$ cubic voxels. As the initial trabecular structure in proximal femur, a random trabecular structure was given by the random-placement of hollow spheres with inside and outside diameters of 2100 and $4200 \mu \mathrm{m}$, respectively (Fig. 6 (a)). One voxel was transferred to one finite element of eight node brick element. The isotropic elastic body with Young's modulus of $20.0 \mathrm{GPa}$ and Poisson's ratio of 0.3 was assumed for trabecular bone material. The cortical outer surface was fixed throughout the remodeling simulation, and the intra-structure, from the head to mid-shaft, was remodeled in accordance with the same remodeling procedure. The fixed boundary conditions are imposed on the distal end. Using the initial structure, we carried out the remodeling simulation under the daily loading condition (Fig. 6(b)) ${ }^{(26)}$. In the daily loading condition, we considered the one-legged stance, abduction, and adduction, which accounted for 60,20 , and $20 \%$ of this condition as illustrated in Fig. 7 . The loading magnitudes and frequencies of these three stances were shown in Table $2^{(19),(21)}$. The extreme ranges of motion of abduction and adduction were assumed. The trabecular structure obtained under this daily loading condition was referred to as the normal structure and also used for the initial structure for the remodeling simulation under reduced weight-bearing conditions.

Two reduced weight-bearing conditions were considered here: infrequent walking and cane-assisted walking. In the infrequent walking condition, we wedged the interval with no loading into the daily loading condition; that is, the infrequent walking condition consisted of unloading and the daily loading conditions (Table 3). The rate of unloading interval changed from 10 to $70 \%$ while the proportions of one-legged stance, abduction, and adduction were remained unchanged. In the cane-assisted walking, we referred to the study on relative changes in muscle activity and kinetics during cane-assisted walking ${ }^{(32)}$. The cane use reduced the demand on the hip abductors and decreased joint compression forces related to muscle contraction in the contralateral side. We calculated joint force and hip abductor force at various rates of leaning force when using a cane (Table 4). That is, in the infrequent walking, the number of loading cycles per day decreased from the daily-loading condition (Table 2) while the same loading forces were applied to the femur; in the cane-assisted walking, the numbers of loading cycles per day remained unchanged but the loading forces were reduced according to the leaning force from the daily-loading condition. 


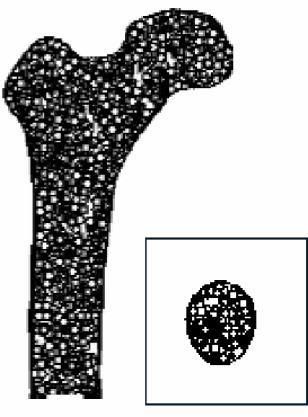

(a) Initial structure

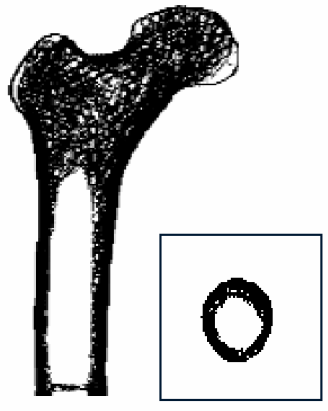

(b) Standard structure

Fig. 6 Profile of human femur was derived on the basis of CT-images.

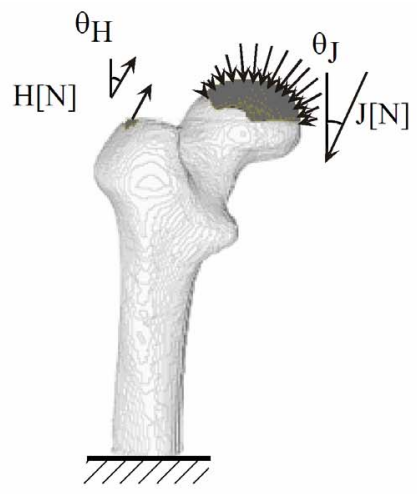

(a) one-legged

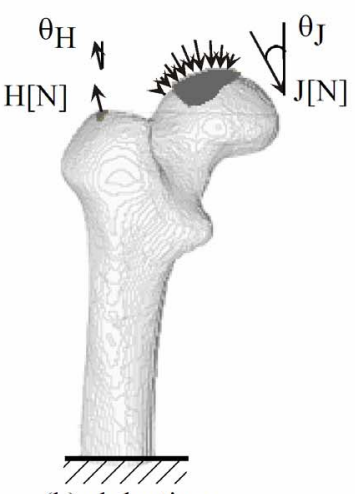

(b) abduction

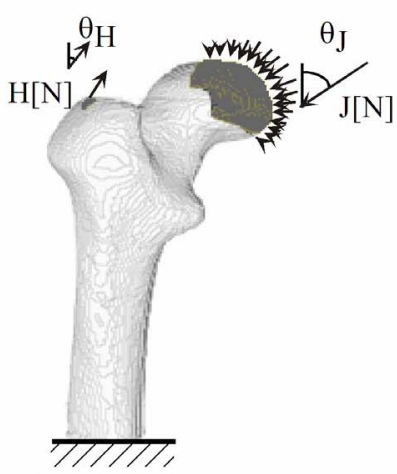

(c) adduction

Fig. 7 Standard weight-bearing cases at each stance (daily-loading condition).

Table 2 Standard magnitude of force and frequency at each stance (daily-loading condition).

\begin{tabular}{c|c|c|c|c|c}
\hline & \multicolumn{2}{|c|}{ Joint reaction force } & \multicolumn{2}{c|}{ Hip abductor force } & \multirow{2}{*}{$\begin{array}{c}\text { Frequency } \\
\text { [cycle/day] }\end{array}$} \\
\cline { 2 - 5 } & $\theta_{\mathrm{J}}$ & $\mathrm{J}[\mathrm{N}]$ & $\theta_{\mathrm{H}}$ & $\mathrm{H}[\mathrm{N}]$ & 6000 \\
\hline One-legged & $24^{\circ}$ & 2317 & $28^{\circ}$ & 703 & 2000 \\
Abduction & $-15^{\circ}$ & 1158 & $-8^{\circ}$ & 351 & 2000 \\
Adduction & $56^{\circ}$ & 1548 & $35^{\circ}$ & 468 & \\
\hline
\end{tabular}

Table 3 Infrequent walking conditions at various unloading rates.

\begin{tabular}{c|c|c|c|c}
\hline $\begin{array}{c}\text { Rate of } \\
\text { unloading }\end{array}$ & $\begin{array}{c}\text { Unloading } \\
\text { [cycle/day] }\end{array}$ & $\begin{array}{c}\text { One-legged } \\
\text { [cycle/day] }\end{array}$ & $\begin{array}{c}\text { Abduction } \\
\text { [cycle/day] }\end{array}$ & $\begin{array}{c}\text { Adduction } \\
\text { [cycle/day] }\end{array}$ \\
\hline $10 \%$ & 1000 & 5400 & 1800 & 1800 \\
$20 \%$ & 2000 & 4800 & 1600 & 1600 \\
$30 \%$ & 3000 & 4200 & 1400 & 1400 \\
$40 \%$ & 4000 & 3600 & 1200 & 1200 \\
$50 \%$ & 5000 & 3000 & 1000 & 1000 \\
$60 \%$ & 6000 & 2400 & 800 & 800 \\
$70 \%$ & 7000 & 1800 & 600 & 600 \\
\hline
\end{tabular}

\footnotetext{
* The interval with no loading is embedded into the daily loading condition as unloading ${ }^{(14)}$.
}

The cycle number corresponds to the interval length of unloading condition. 
Table 4 Cane-assisted walking condition with various rates of leaning force.

\begin{tabular}{c|c|c|c|c|c|c}
\hline \multirow{2}{*}{$\begin{array}{c}\text { Leaning force } \\
\text { using a cane }\end{array}$} & \multicolumn{2}{|c|}{ One-legged } & \multicolumn{2}{c|}{ Abduction } & \multicolumn{2}{c}{ Adduction } \\
\cline { 2 - 7 } & $\mathrm{J}[\mathrm{N}]$ & $\mathrm{H}[\mathrm{N}]$ & $\mathrm{J}[\mathrm{N}]$ & $\mathrm{H}[\mathrm{N}]$ & $\mathrm{J}[\mathrm{N}]$ & $\mathrm{H}[\mathrm{N}]$ \\
\hline $0 \%$ & 2317 & 703 & 1158 & 351 & 1548 & 468 \\
$10 \%$ & 1121 & 340 & 560 & 170 & 749 & 227 \\
$20 \%$ & 1229 & 283 & 614 & 141 & 821 & 189 \\
$30 \%$ & 1341 & 225 & 670 & 113 & 896 & 150 \\
$40 \%$ & 1456 & 167 & 728 & 84 & 973 & 111 \\
$50 \%$ & 1572 & 109 & 786 & 55 & 1050 & 73 \\
$60 \%$ & 1690 & 51 & 845 & 26 & 1129 & 34 \\
$70 \%$ & 1809 & 7 & 904 & 3 & 1209 & 4 \\
\hline
\end{tabular}

\section{Results}

The trabecular structure of the human proximal femur under the infrequent walking condition was shown for each rate of unloading interval and the initial, i.e., normal trabecular structure was also shown in Fig. 8. Initial structure resembles to that the observed in the cross sectional pattern diagram of normal human femur, as shown in Fig. 9. In the initial structure, all the normal groups of trabecular are visible; the compressive and tensile trabecular bones cross each other and the upper end of femur is completely occupied by cancellous tissue. Even Ward's triangle shows some thin trabecular bone ${ }^{(33)}$.

Increasing the rate of unloading interval led to the increased bone loss, first in the femoral head and the greater trochanter. Ward's triangle was emptier in the higher rate of unloading frequency. Subsequently, the secondary compressive group disappeared and the principal tensile group became thinner with further increasing of the rate of unloading. When unloading level increased up to $70 \%$, the principal compressive group little remained although the greater trochanter group was still observed clearly.

Figure 10 shows the trabecular structure changes under cane-assisted walking conditions. Using cane decreased joint compression forces related to muscle contraction in the contralateral side (Table 4). Bone loss attracts attention on the greater trochanter group and Ward's triangle became clear when reducing hip abductor forces. Similarly as in Fig. 8, subsequent disappearing of the secondary compressive group and the thinning of the principal tensile group were observed with further increasing of the rate of unloading. However, bone loss in the trabecular femoral head differed from that under the infrequent walking condition. The hip joint load will be involved in this difference.

Clinically, the correlation of bed rest (reducing loading frequency) and muscle activity has been reported. The disuse of bed rest with diseases accompanies with reduced muscle activity, and then leads to the cane use for walking. Thus, we simulated the trabecular remodeling by integrating the two reduced weight-bearing conditions. Figure 11 shows that trabecular loss increased with increasing in the rate of unloading interval. Combination of reductions of loading frequency and loading forces relatively accentuated the structure of principal compressive and principal tensile groups and enhanced the thinning of the secondary compressive trabecular bone. Therefore, empty Ward's triangle is clearly observed. Further increase in the rate of unloading interval led to a marked reduction of tensile trabecular bone and discontinued the principal tensile group. Thus, the tensile trabecular bone is observed only in the upper part of the femoral head, where trabeculae are still comparable in density to the principal compressive trabeculae. Finally, even the principal compressive group became less obvious. 


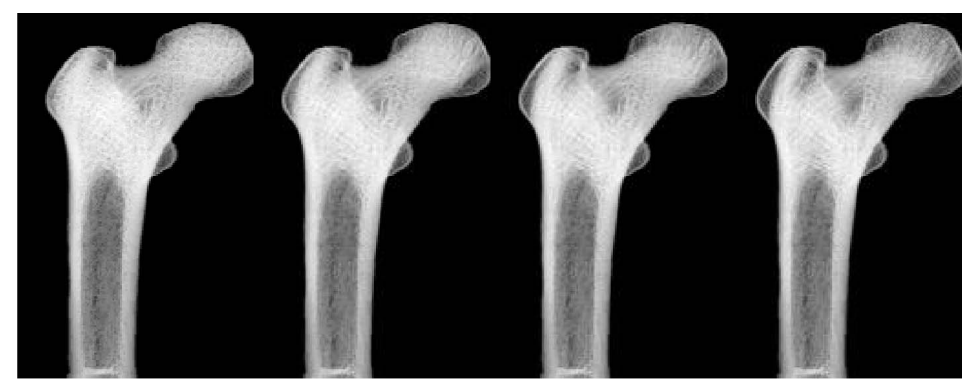

(a) Initial structure

(b) $10 \%$

(c) $20 \%$

(d) $30 \%$
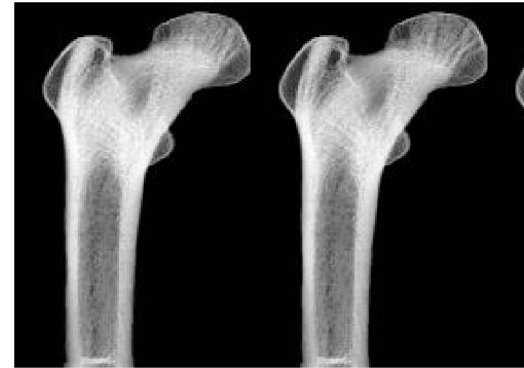

(e) $40 \%$

(f) $50 \%$

(g) $60 \%$

(h) $70 \%$

Fig. 8 Trabecular structure predicted by remodeling simulation under infrequent walking conditions.

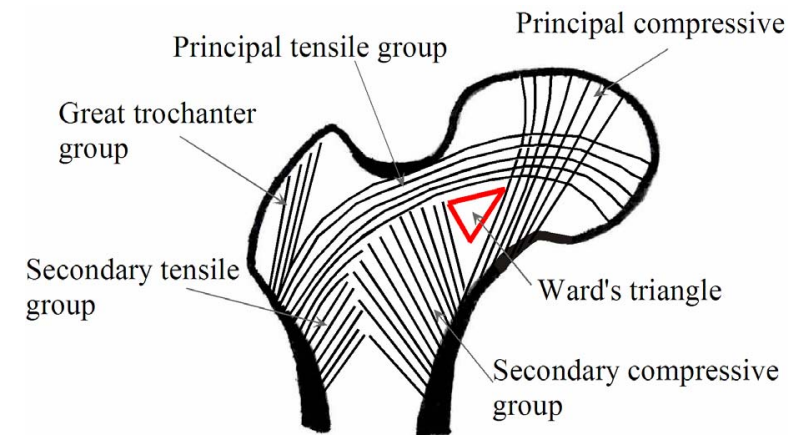

Fig. 9 Pattern diagram of trabecular structure of human femur.



(a) Initial structure $\quad$ (b) $10 \%$

(c) $20 \%$

(d) $30 \%$

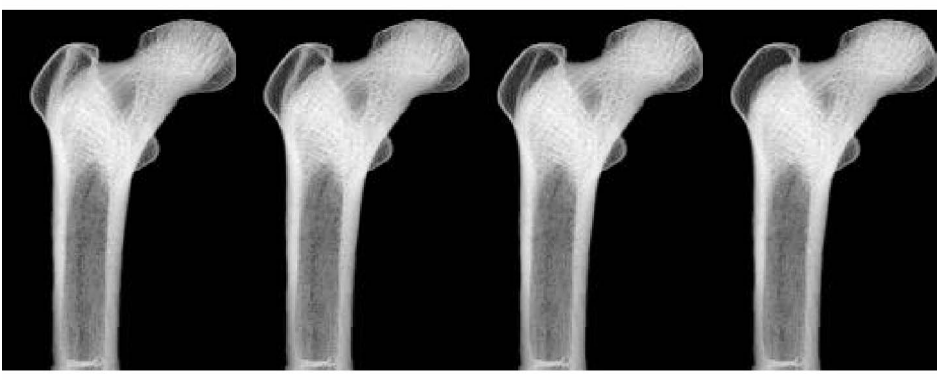

(e) $40 \%$

(f) $50 \%$

(g) $60 \%$

(h) $70 \%$

Fig. 10 Trabecular structure predicted by remodeling simulation under cane-assisted walking conditions. 


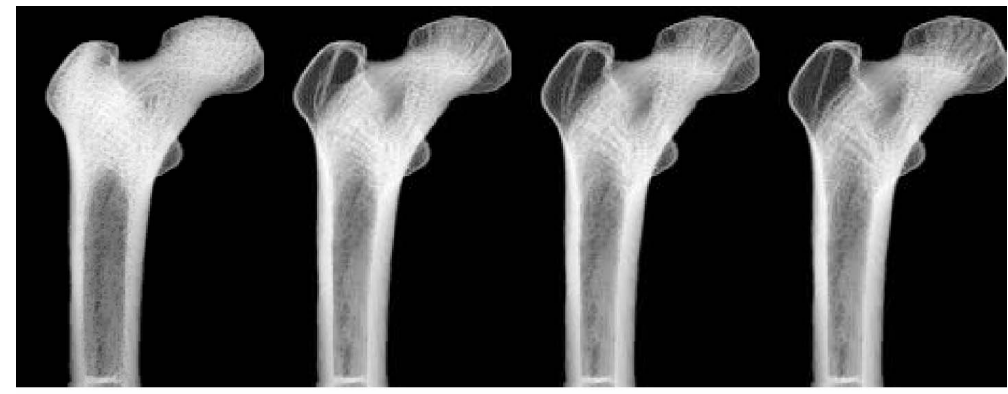

(a) Initial structure $\quad$ (b) $10 \%$

(c) $20 \%$

(d) $30 \%$

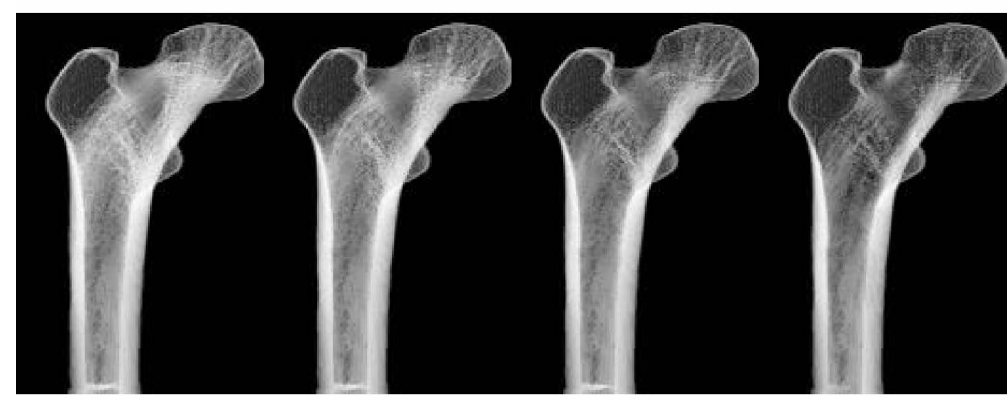

(e) $40 \%$

(f) $50 \%$

(g) $60 \%$

(h) $70 \%$

Fig. 11 Trabecular structure predicted by remodeling simulation at various rates of unloading interval (the integrated reduced weight-bearing condition).

\section{Discussion}

Trabecular structure of the proximal femur is remodeled differently in response to various forms of mechanical stimulation ${ }^{(1),(2)}$. In this study, we investigated that the structure changes of human trabecular bone under the reduced weight-bearing conditions by a surface remodeling model ${ }^{(26)}$, in which osteocyte apoptosis plays a crucial role below a physiological strain range ${ }^{(28)}$. The reduced weight-bearing conditions were imposed by assuming infrequent or cane-assisted walking. Depending on the reduced weight-bearing conditions, the trabecular structure reached different equilibrium structures even if the same initial structure and model parameters are used. In all cases, trabecular bone loss occurred in relation to the mechanical stimuli, although there was a regional difference in the pattern of bone loss between the two conditions. In the infrequent and cane-assisted walking conditions, significant bone loss occurred in the great trochanter and in the femoral head, respectively.

In the results of imposing the condition combining two reduced weight-bearing conditions, we found out the clinically observed trabecular structure in osteoporotic human proximal femur. The description for degree of osteoporotic trabecular structure (Singh Index) is shown in Table 5. The present simulations showed that decreasing mechanical stimuli enhanced the degree of osteoporosis along with the grade defined by Singh et al ${ }^{(33)}$. Figure 11 shows trabecular loss at various degrees of unloading. In the initial structure (Fig. 11(a)), all groups of trabecular bones are visible, which corresponds to Grade 6 in Singh index (Table 5). The compressive and tensile trabeculae intersect each other and the upper end of the femur is completely occupied by cancellous rich structure. Ward's triangle is not clearly delineated and there are some thin trabeculae in it. With increasing the proportion of unloading, there occurs an apparent accentuation of the structure of the principal compressive and principal tensile groups, while the secondary compressive trabeculae became thinner (Grade 5, Fig. 11(b) (c)). As a result, an empty region appears in Ward's triangle. Further increase in the proportion of unloading leads to the marked reduction in the tensile trabecular bones (Grade 4, Fig. 11(d) (e)); in due course, discontinuity occurs in the 
principal tensile group. At the stages of Fig. 11(e) and (f), the tensile trabeculae are seen only in the upper part of the femoral head, where the trabeculae are still comparable in density to the principal compressive trabeculae (Grade 3). Finally, even the principal compressive group ceases to stand out (Grade 2, Fig. 11(g)) and decreases markedly in number (Grade 1, Fig. 11(h)). Therefore, the present remodeling model may be used to study the trabecular bone loss in disuse-mediated osteoporosis. Bone resorption-dominant remodeling, introduced into the present model to consider the effect of osteocyte apoptosis under low strain stimuli, may be essential in predicting the osteoporotic change of trabecular bone structure.

Finite element analyses were conducted for normal and osteoporotic human femurs obtained by remodeling simulation, and trabecular bone stress and strain were examined for a spherical volume of interest in the femoral head ${ }^{(34)}$. Figure 12 shows the distribution of voxel at different stress/strain for the normal femur of Fig. 11(a) and an osteoporotic femur of Fig. 11(b) that is obtained under the disuse loading condition of reduced loading frequency and force. Solid and broken blue lines distinguish the stress/strain distribution for osteoporotic femur under standard and disuse loading conditions, respectively. Trabecular bone stress and strain in the osteoporotic femur were distributed more uniformly than those in the normal femur. In the osteoporotic femur, trabecular bones orthogonal to the alignment of the principal compressive group (non-principal trabeculae) are relatively-scarce because osteocytes in those trabeculae, especially in non-principal group originally exposed to relatively small loading, are susceptible to apoptosis when subjected to the disuse condition, resulting in scarcely distributed non-principal trabeculae. Consequently, trabeculae undergoing relatively-low stress/strain decrease and the peaks of stress and strain distributions increased in the osteoporotic femur. Trabeculae in principal compressive group are likely to preserve their role of relatively high load-bearing even under the disuse condition.

Table 5 Singh Index

\begin{tabular}{c|l}
\hline Grade 6 & $\begin{array}{l}\text { All the normal trabecular groups are visible and the upper end of the femur } \\
\text { seems to be completely occupied by trabecular bone. }\end{array}$ \\
\hline Grade 5 & $\begin{array}{l}\text { The structure of principal tensile and principal compressive trabeculae is } \\
\text { accentuated. Ward's triangle appears prominent. }\end{array}$ \\
\hline Grade 4 & $\begin{array}{l}\text { Principal tensile trabeculae are markedly reduced in number but can still be } \\
\text { observed in lateral cortex to the upper part of the femoral neck. }\end{array}$ \\
\hline Grade 3 & $\begin{array}{l}\text { There is a break in the continuity of the principal tensile trabeculae opposite } \\
\text { the greater trochanter. This grade indicates definite osteoporosis. }\end{array}$ \\
\hline Grade 2 & $\begin{array}{l}\text { Only the principal compressive trabeculae stand out prominently; the others } \\
\text { have been resorbed more or less completely. }\end{array}$ \\
\hline Grade 1 & $\begin{array}{l}\text { Even the principal compressive trabeculae are markedly reduced in number and } \\
\text { are no longer prominent. }\end{array}$ \\
\hline
\end{tabular}

\section{Conclusion}

A mathematical model of trabecular bone remodeling, which could account for disuse-accelerated bone loss and overuse-mediated targeted remodeling, was used for predicting the changes of trabecular bone structure in human femur under infrequent and cane-assisted walking condition. Compared with the structure derived under normal daily 


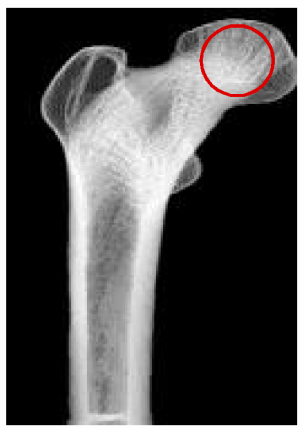

(a) Osteoporotic femur(Fig.11 (b)) Red circle indicates a spherical volume of interest.

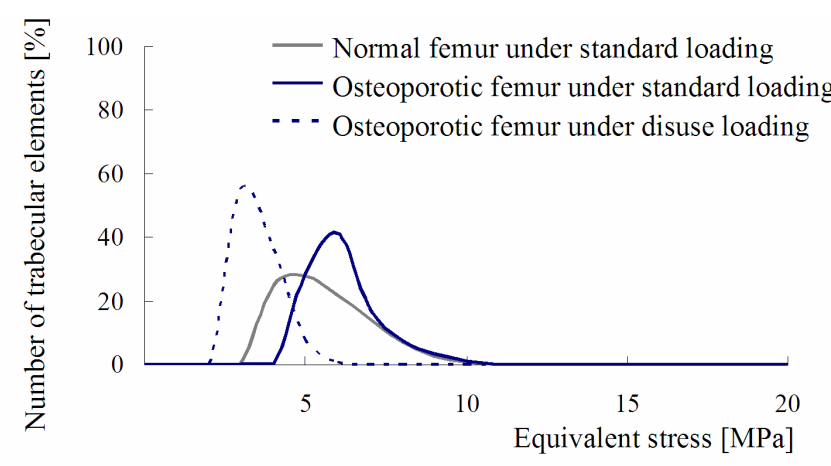

(b) Equivalent stress in ROI



(c) Equivalent strain in ROI

Fig. 12 Equivalent stress and strain distributions in normal and osteoporotic femurs.

loading condition, trabecular bone reduced with increased degree of disuse in a manner consistent to Singh Index, diagnostic criterion for the stage of osteoporosis progression. Thus, the present remodeling model is effective in bone remodeling simulation under reduced loading condition.

\section{References}

(1) Wolff, J., The Law of Bone Remodeling, Springer (Trans. Maquet, P., Furlong, R.), (1986).

(2) Carter, D.R., Mechanical loading history and skeletal biology, Journal of Biomechanics, Vol. 20 (1987), pp. 1095-1109.

(3) Parfitt, A.M., Osteonal and hemi-osteonal remodeling: the spatial and temporal framework for signal traffic in adult human bone, Journal of Cellular Biochemistry, Vol. 55 (1994), pp. 273-286.

(4) Duncan, R.L., Turner, C.H., Mechanotransduction and the functional response of bone to mechanical strain, Calcified Tissue International, Vol. 57, No. 5 (1995), pp. 344-358.

(5) Vico, L., Collet, A., Guignandon, P., Lafage-Proust, M.H., Thomas, T., Rehailia, M., Alexandre, C., Effects of long term microgravity exposure on cancellous and cortical weight-bearing bones of cosmonauts, The Lancet, Vol. 355 (2000), pp. 1607-1611.

(6) Lang, T., LeBlanc, A., Evans, H., Lu, Y., Genant, H., Yu, A., Cortical and trabecular bone mineral loss from the spine and hip in long-duration spaceflight, Journal of Bone Mineral Research, Vol. 19 (2004), pp. 1006-1012. 
(7) Takata, S., Yasui, N., Disuse osteoporosis, The Journal of Medical Investigation, Vol. 48 (2001), pp. 147-156.

(8) Shackelford, L.C., LeBlanc, A.D., Driscoll, T.B., Evans, H.J., Rianon, N.J., Smith, S.M., Spector, E., Feeback, D.L., Lai, D., Resistance exercise as a countermeasure to disuse-induced bone loss, Journal of Applied Physiology, Vol. 97 (2004), pp. 119-129.

(9) LeBlanc, A.D., Schneider, V.S., Evans, H.J., Engelbretson, D.A., Krebs, J.M., Bone mineral loss and recovery after 17 weeks of bed rest, Journal of Bone and Mineral Research, Vol. 5 (1990), pp. 843-850.

(10) Barrera, G., Bunout, D., Gattás, V., Maza, M.P., Leiva, L., Hirsch, S., A high body mass index protects against femoral neck osteoporosis in healthy elderly subjects, Nutrition, Vol. 20, No. 9 (2004), pp. 769-771.

(11) Sordia, L.H., Vazquez, J., Iglesias, J.L., Piñeyro, M.O., Vidal, O., Saldivar, D., Morales A., Merino, M. Pons, G., Rosales, E., Low height and low weight correlates better with osteoporosis than low body mass index in postmenopausal woman, International Congress Series, Vol. 1271 (2004), pp. 407-410.

(12) Vailas, A.C., Zernicke, R.F., Grindeland, R.E., Kaplansky, A., Durnova, G.N., Li, K.C., Martinez, D.A., Effects of spaceflight on rat humerus geometry, biomechanics, and biochemistry. The FASEB Journal, Vol. 4 (1990), pp. 47-54.

(13) Garber, M.A., McDowell, D.L., Hutton, W.C., Bone loss during simulated weightlessness: a biomechanical and mineralization study in the rat model, Aviation Space Environmental Medicine, Vol. 71 (2000), pp. 586-592.

(14) Baiotto, S., Labat B., Vico, L., Zidi, M., Bone remodeling regulation under unloading condition: Numerical investigations, Computer in Biology and Medicine, Vol. 39 (2009), pp. 46-52.

(15)Kaneps, A.J., Stover, S.M., Lane, N.E., Changes in canine cortical and cancellous bone mechanical properties following immobilization and remobilization with exercise, Bone, Vol. 21 (1997), pp. 419-423.

(16)Li, C.Y., Majeska, R.J., Laudier, D.M., Mann, R., Schaffler, M.B., High-dose risedronate treatment partially preserves cancellous bone mass and microarchitecture during long-term disuse, Bone, Vol. 37 (2005), pp. 287-295.

(17)Frost, H.M., Bone's mechanostat: a 2003 update, The Anatomical Record, Part A, Discoveries in molecular, cellular, and evolutionary biology, Vol. 275, No. 2 (2003), pp. 1081-1101.

(18) Ruimerman, R., Huiskes, R., Van Lenthe, G.H., Janssen, J.D., A computer simulation model relating bone-cell metabolism to mechanical adaptation of trabecular bone, Computational Methods in Biomechanical and Biomedical Engineering, Vol. 4 (2001), pp. 433-448.

(19) Beaupré, G.S., Orr, T.E., Carter, D.R., An approach for time-dependent bone modeling-application: a preliminary remodeling simulation, Journal of Orthopedic Research, Vol. 8 (1990), pp. 662-670.

(20)Luo, G., Cowin, S.C., Sadegh, A.M., Arramon, Y.P., Implementation of strain rate as a bone remodeling stimulus, Journal of Biomechanical Engineering, Vol. 117 (1995), pp. 329-338.

(21) Adachi, T., Tomita, Y., Sakaue, H., Tanaka, M., Simulation of trabecular surface remodeling based on local stress nonuniformity, JSME International Journal, Series C, Vol. 40, No. 4 (1997), pp. 782-792.

(22) Adachi, T., Tsubota, K., Tomita, Y., Hollister, S.J., Trabecular surface remodeling simulation for cancellous bone using microstructural voxel finite element models, Journal of Biomechanical Engineering, Vol. 123 (2001), pp. 403-409. 
(23) Tsubota, K., Adachi, T., Tomita, Y., Functional adaptation of cancellous bone in human proximal femur predicted by trabecular surface remodeling simulation toward uniform stress state, Journal of Biomechanics, Vol. 35 (2002), pp. 1541-1551.

(24) Tsubota, K., Adachi, T., Simulation study on local and integral mechanical quantities at single trabecular level as candidate of remodeling stimuli, Journal of Biomechanical Science and Engineering, Vol. 1 (2006), pp. 124-135.

(25) Tsubota, K., Suzuki, Y., Yamada, T., Hojo, M., Makinouchi, M., Adachi, T., Computer simulation of trabecular remodeling in human proximal femur using large-scale voxel FE models: Approach to understanding Wolff's law, Journal of Biomechanics, Vol. 42 (2009), pp. 1088-1094.

(26) Kwon, J.Y., Naito, H., Matsumoto, T., Tanaka, M., Simulation model of trabecular bone remodeling considering effects of osteocytes apoptosis and targeted remodeling, Journal of Biomechanical Science and Engineering, Vol. 5, No. 5 (2010), pp. 539-551.

(27) Noble, B.S., Peet, N., Stevens, H.Y., Brabbs, A., Mosley, J.R., Reilly, G.C., Reeve, J., Skerry, T.M., Lanyon, L.E., Mechanical loading: biphasic osteocyte survival and targeting of osteoclasts for bone destruction in rat cortical bone, American Physiological Society, Vol. 284, No. 4 (2003), pp. C934-C943.

(28) Gu, G., Mulari, M., Peng, Z., Hentuen, T.A., Väänänen, H.K., Death of osteocytes turns off the inhabitation of osteoclasts and triggers local bone resorption, Biomechanical and Biophysical Research Communications, Vol. 335, No. 4 (2005), pp. 1095-1101.

(29)Li, C.Y., Majeska, R.J., Laudier, D.M., Mann, R., Schaffler, M.B., High-dose risedronate treatment partially preserves cancellous bone mass and microarchitecture during long-term disuse, Bone, Vol. 37, No. 3 (2005), pp. 287-295.

(30) Burr, D.B., Targeted and nontargeted remodeling, Bone, Vol. 30, No. 1 (2002), pp. 2-4.

(31) Da Costa Gómez, T.M., Barrett, J.G., Sample, S.J., Radtke, C.L., Kalscheur, V.L., Lu, Y., Markel, M.D., Santschi, E.M., Scollay, M.C., Muir, P., Up-regulation of site-specific remodeling without accumulation of microcracking and loss of osteocytes, Bone, Vol. 37, No. 1 (2005), pp. 16-24.

(32) Neumann, D.A., Hip abductor muscle activity as subjects with hip prostheses walk with different methods of using a cane, Physical Therapy, Vol. 78 (1998), pp. 490-501.

(33) Singh, M., Nagrath, A.R., Maini, P.S., Changes in Trabecular Pattern of the Upper end of the Femur as an index of osteoporosis, The Journal of Bone and Joint Surgery, Vol. 52 (1970), pp. 457-467.

(34) Rietbergen, B., Huiskes, R., Eckstein, F., Rüegsegger, P., Trabecular bone tissue strains in the healthy and osteoporotic human femur, Journal of Bone and Mineral Research, Vol. 18, No. 10 (2003), pp. 1781-1788. 